\title{
Preface
}

\section{FDG-PET/CT: Whither I Go, \\ Thou Canst Not Follow Me \\ Now; but Thou Shalt Follow \\ Me Afterwards}

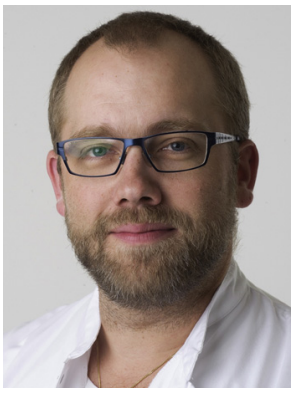

Søren Hess, MD

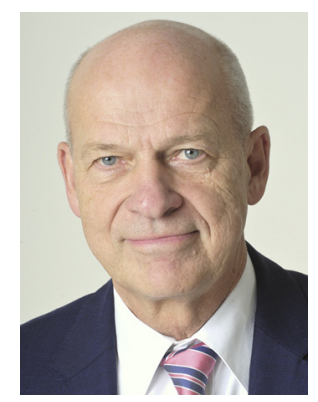

Poul Flemming Høilund-Carlsen, MD, DMSc Editors
According to history, the quotation in the title above is one of two answers provided to the famous words, quo vadis, uttered by Jesus to his disciple Peter and provocatively inquired by us in our introduction to the first of two issues of $P E T$ Clinics on the current use of FDG-PET/CT. ${ }^{1}$ Without sounding too pompous, the very same words may well be used in response to the question of where FDG-PET/CT is going. The modality PET has been around for almost 40 years; the predominant PET tracer FDG has been known since $1976,{ }^{2}$ and hybrid PET/CT has been commercially available since the millennium. Yet, doubts remain with regard to the method's potential and possible superiority in various clinical settings. Perhaps, this is not too surprising considering how few randomized studies actually exist on the method's clinical value, ${ }^{3}$ not to mention the few economic evaluations of the use of PET or PET/CT that can be found in the literature; regarding the latter, please see the article by Oke Gerke and coworkers in the present issue of PET Clinics.

What can be done? The first step is to record and register the type and quality of evidence available so far. The next step would be to identify the shortcomings of the existing documentation and pinpoint what clinical areas are potential fields for a more extensive and focused use of FDG-PET and FDG-PET/CT imaging and which questions should be answered satisfactorily to justify the use of PET as an adjunct for or a substitute of existing methodologies.

In line with this, the present part II of a series of articles focusing on the clinical use of FDG-PET and, in particular, modern FDG-PET/CT imaging in a variety of diseases aims to explore how far the methodology has reached and pinpoint areas in which documentation is sparse and further studies are needed. Thus, Eivind Segtnan and colleagues present the current status on the use of FDG in primary brain tumors, the first application of positron emission imaging, and one of the earliest clinical applications of FDG. The potential remains significant despite inherent difficulties with visualizing pathology against the high physiologic FDG uptake in the brain. Jeppe Lauridsen and colleagues provide an overview of FDG-PET and FDG-PET/CT in head and neck malignancies, including thyroid cancer, anatomically close but still very different disease entities. Thus, head and neck squamous cell carcinoma is an important and common indication in most centers, especially for initial staging where FDG imaging is clearly superior to conventional imaging. And whereas FDG has definite potential in differentiated thyroid cancers, its definite role in other subtypes remains unresolved. The same pattern goes for the female-specific malignancies presented by 
Malene Hildebrandt and colleagues: there is variable potential for the four most frequent female-specific cancers (ie, breast, endometrial, ovarian, and cervical cancer) in staging, prognostication, response evaluation, recurrence detection, and radiation therapy planning, but to quote the authors, the evidence points toward a "technique under constant development." More thorough studies and documentation are warranted to properly define the clinical application and value of FDG and PET in these settings. Outside the realm of cancer, Björn Blomberg and HøilundCarlsen outline an emerging application of FDG, namely, imaging of atherosclerosis, which could provide valuable insights into the pathophysiology of one of the world's leading causes of morbidity and mortality. By targeting atherosclerotic plaque glycolysis, FDG may serve as a marker for plaque inflammation and hypoxia. As such, FDG can be a potential surrogate endpoint in drug trials, can provide prognostic information superior to traditional cardiovascular risk scores, and perhaps may be able to identify the rupture-prone plaques considered by many to be the single most important risk factor for cardiac events. Two articles deal with the use of FDG in planning and assessment of cancer therapy. Thus, Christina Speirs and colleagues provide an overview of the literature on the use of FDG-PET and FDG-PET/CT in radiation treatment planning, which is still primarily based on morphologic imaging. However, by adding metabolic information to anatomical gross target volumes and clinical target volumes, FDG offers better target delineation, including involved lymph nodes. In that respect, FDG-PET and FDG-PET/ CT may assist in maximizing the radiation effect with the least collateral damage as well as may enable dose fractioning according to biologic target volumes, so-called dose painting. Finally, Sandip Basu and colleagues evaluate the evolution of various response assessment criteria. Objective and reliable evaluation of treatment response is pivotal in cancer management in order to personalize treatment strategy (ie, separate patients benefiting from a certain treatment from those not benefiting). Conventional response assessment by morphologic changes may lag behind actual biologic response and underestimate response. Functional imaging may provide substantially earlier evidence of response, and FDG imaging is likely to be the diagnostic modality of choice for assessing treatment response in cancer and systemic inflammatory disorders.

An important issue of FDG imaging is novel quantitative techniques. A special article by Sina Houshmand and colleagues highlights exactly this unique property of PET, its ability to provide quantitative information of the individual patient's disease, before, during, and after therapy, be it in the shape of a single number indicating the state of a focal process or as a "global disease score" that reflects the sum of active disease in the body, which may serve as guidance for assessment of treatment efficacy. This is of major importance, since these options are not offered by any other imaging modality, which at best can only provide semiquantitative grading based on visual analysis, which eo ipso is observer-dependent. However, even PET has stagnated in recording easily obtainable quantitative parameters, like the standard uptake value that has gained popularity as it is automatically read out by any PET scanner. Its frequent use and potential relation to clinical conditions and outcomes have blinded many users and obscured the fact that these values may at times be more misleading than instructive, simply because they are not recorded and interpreted the way they should. Proper achievement requires precise tissue segmentation and corrections, including correction for partial volume effect, and these circumstances will significantly change the size of the parameter and, hence, paint a completely different and more accurate picture of the disease than what can be deduced from the popular automatically derived values that at best are no more than proxy indicators.

Thus, referring to the title, to follow the road of PET has for some decades been a cumbersome and prickly travel. However, thanks to substantial improvements of scanner and computer technology, the time has come to prepare the way for PET to become the most important clinical imaging modality of the twenty-first century. Nothing can replace the opportunities that lie in being able to identify specific molecules and their presence, location, and quantity in the body as an expression of ongoing, active or dormant, disease. Those who understand the key role of this type of information will get on the move and bring medicine a step further. Structural imaging and analyses of tissue, blood, and other body fluids will help and be indicative, but cannot deliver the type and amount of essential knowledge provided by PET and, in particular, FDG-PET and FDG-PET/CT.

Søren Hess, MD Department of Nuclear Medicine Odense University Hospital Soender Boulevard 29 5000 Odense, Denmark 
Poul Flemming Høilund-Carlsen, MD, DMSc Department of Nuclear Medicine Odense University Hospital Institute of Clinical Research University of Southern Denmark

Soender Boulevard 29 5000 Odense, Denmark

E-mail addresses: soeren.hess@rsyd.dk (S. Hess) pfhc@rsyd.dk (P.F. Høilund-Carlsen)

\section{REFERENCES}

1. Hess S, Høilund-Carlsen PF. FDG-PET/CT: quo vadis? PET Clin 2014;9(4):xi-xiii.

2. Reivich M, Kuhl D, Wolf A, et al. Measurement of local cerebral glucose metabolism in man with 18F-2-fluoro-2-deoxy-d-glucose. Acta Neurol Scand Suppl 1977;64:190-1.

3. Siepe B, Hoilund-Carlsen PF, Gerke O, et al. The move from accuracy studies to randomized trials in PET: current status and future directions. I Nucl Med 2014;55:1228-34. 